Homology, Homotopy and Applications, vol.20(2), 2018, pp.1-10

\title{
DECOMPOSING MANIFOLDS INTO CARTESIAN PRODUCTS
}

\author{
SLAWOMIR KWASIK AND REINHARD SCHULTZ
}

(communicated by Jonathan M. Rosenberg)

\begin{abstract}
The decomposability of a Cartesian product of two nondecomposable manifolds into products of lower dimensional manifolds is studied. For 3-manifolds we obtain an analog of a result due to Borsuk for surfaces, and in higher dimensions we show that similar analogs do not exist unless one imposes further restrictions such as simple connectivity.
\end{abstract}

\section{Introduction}

There are plenty of examples which show the nonuniqueness of splitting a space (manifold) into Cartesian products. For example, there is the well known Bing space, a generalized 3-manifold $X$ (cf. [Bi]) such that $X \neq \mathbb{R}^{3}$ and $X \times \mathbb{R}=\mathbb{R}^{4}=\mathbb{R}^{3} \times \mathbb{R}$, or the open 3 -manifold $\mathcal{W}$ of Whitehead ( $f$. $[\mathbf{H e}]$ ), where $\mathcal{W} \neq \mathbb{R}^{3}$ and again $\mathcal{W} \times \mathbb{R}=$ $\mathbb{R}^{4}=\mathbb{R}^{3} \times \mathbb{R}$ (here $=$ stands for homeomorphic). More dramatic examples are pairs of two homotopy inequivalent 3-dimensional Seifert manifolds $\mathcal{M}$ and $\mathcal{N}$ such that $\mathcal{M} \times S^{1}=\mathcal{N} \times S^{1}(c f .[\mathbf{C R}, \mathbf{K R 2}])$.

However, on the positive side there is an old result of Borsuk ( $c f$. [Bo]) that a closed, $n$-dimensional manifold has at most one decomposition into the Cartesian product of indecomposable factors of dimension $\leqslant 2$.

Now suppose we that have two closed, oriented $n$-dimensional manifolds $\mathcal{M}^{n}$ and $\mathcal{N}^{n}$ which cannot be split into products of closed, oriented manifolds $(\neq\{\mathrm{pt}\})$ of lower dimension. Here is one natural question: $\operatorname{Can} \mathcal{M}^{n} \times \mathcal{N}^{n}$ be decomposed into products of manifolds of dimension $\leqslant n-1$ ?

More generally, we shall consider the following situation (we recall that we are working in the TOP category, i.e., topological manifolds and homeomorphisms):

Let $\mathcal{M}^{n}, \mathcal{N}^{k}$ be closed, oriented, indecomposable (into nontrivial Cartesian products) manifolds of dimension $n$ and $k$ respectively, $k \leqslant n$. One says that the manifold $\mathcal{N}^{k}$ stably decomposes $\mathcal{M}^{n}$ if $\mathcal{M}^{n} \times \mathcal{N}^{k}$ can be written as a Cartesian product of manifolds of dimension $\leqslant n-1$ (i.e. $\mathcal{M}^{n} \times \mathcal{N}^{k}=\prod_{i} Y_{i}^{n_{i}}$ such that each $Y_{i}^{n_{i}}$ is a closed manifold of dimension $n_{i}$, where $1 \leqslant n_{i} \leqslant n-1$ and $\sum_{i} n_{i}=n+k$ ). If for a given manifold $\mathcal{M}^{n}$, there is no such $\mathcal{N}^{k}$, then $\mathcal{M}^{n}$ is called stably nondecomposable.

Received May 25, 2017, revised November 29, 2017; published on March 20, 2018.

2010 Mathematics Subject Classification: 57R80, 57M50.

Key words and phrases: Seifert manifold, Whitehead torsion, s-cobordism, surgery group.

Article available at http://dx.doi.org/10.4310/HHA.2018.v20.n2.a1

Copyright (C) 2018, Slawomir Kwasik and Reinhard Schultz. Permission to copy for private use granted. 
If $n=1$ or $n=2$, then Borsuk's result shows that every $\mathcal{M}^{n}$ is stably nondecomposable. It turns out that this is also true for $n=3$ :

Theorem 1.1. Let $\mathcal{M}^{3}$ be an oriented, closed, nondecomposable 3-manifold. Then $\mathcal{M}^{3}$ is stably nondecomposable.

On the other hand, for $n=4$ we have the following:

Theorem 1.2. There exists an oriented, closed, nondecomposable 4-manifold $\mathcal{M}^{4}$ such that $\mathcal{M}^{4} \times S^{k}=S^{1} \times S^{k} \times \mathbb{R P}^{3}(k=2,3,4)$. Moreover there are infinitely many non-decomposable 4-manifolds $\mathcal{M}_{i}^{4}(i=1,2, \ldots, n, \ldots)$ with $\mathcal{M}_{i}^{4} \neq \mathcal{M}_{j}^{4}, i \neq j$ and $\mathcal{M}^{4} \times S^{k}=S^{1} \times S^{k} \times \mathbb{R P}^{3}(k=2,3,4)$.

The manifold $\mathcal{M}^{4}$ in the above theorem is not simply connected. It turns out that this is an essential condition in our proof. Indeed, for simply connected 4-manifolds we have the following addendum to Theorem 1.2.

Theorem 1.3. Let $\mathcal{M}^{4}$ be a closed, simply connected nondecomposable manifold. Then $S^{k}(k=2,3,4)$ cannot stably decompose $\mathcal{M}^{4}$.

\section{Proofs}

This section contains proofs of our results. The methods and techniques employed in these proofs form a curious combination of high-dimensional surgery theory and low-dimensional topology.

Proof of Theorem 1.1. We first consider the case of $\mathcal{M}^{3}$ and $\mathcal{N}^{3}$. Let $\mathcal{M}^{3}$ and $\mathcal{N}^{3}$ be oriented, closed, nondecomposable 3-manifolds.

Suppose $\mathcal{M}^{3} \times \mathcal{N}^{3}$ is decomposable, so we can write $\mathcal{M}^{3} \times \mathcal{N}^{3}=S_{1} \times S_{2} \times S_{3}$, where $\operatorname{dim} S_{i}=2(i=1,2,3)$.

Our first observation is that without loss of generality we can assume $\pi_{1}\left(\mathcal{M}^{3}\right)$, $\pi_{1}\left(\mathcal{N}^{3}\right)$ are infinite. Our second observation is that because the Euler characteristic $\chi\left(\mathcal{M}^{3}\right)=\chi\left(\mathcal{N}^{3}\right)=0$, then at least one of the $S_{i}(i=1,2,3)$ must be a torus $T^{2}=$ $S^{1} \times S^{1}$.

Suppose that exactly one of the $S_{i}$ (say $S_{3}$ ) is a torus. Then $\mathcal{M}^{3} \times \mathcal{N}^{3}=S_{1} \times$ $S_{2} \times S^{1} \times S^{1}$. It follows that the center $C\left(\pi_{1}\left(\mathcal{M}^{3} \times \mathcal{N}^{3}\right)\right)$ of $\pi_{1}\left(\mathcal{M}^{3} \times \mathcal{N}^{3}\right)$ is given by $C\left(\pi_{1}\left(\mathcal{M}^{3} \times \mathcal{N}^{3}\right)\right) \cong C\left(\pi_{1}\left(\mathcal{M}^{3}\right)\right) \oplus C\left(\pi_{1}\left(\mathcal{N}^{3}\right)\right) \cong \mathbb{Z} \oplus \mathbb{Z}$.

Now suppose that the center of $\pi_{1}\left(\mathcal{M}^{3}\right)$ or the center of $\pi_{1}\left(\mathcal{N}^{3}\right)$ is trivial, say $C\left(\pi_{1}\left(\mathcal{M}^{3}\right)\right) \cong 0$. Consequently, $C\left(\pi_{1}\left(\mathcal{N}^{3}\right)\right) \cong \mathbb{Z} \oplus \mathbb{Z}$. This implies that $\mathcal{N}^{3}=S^{1} \times$ $S^{1} \times S^{1}$ (i.e., Theorem 12.10, p. 131 in $[\mathbf{H e}]$ ), and we are done.

Assume now that $C\left(\pi_{1}\left(\mathcal{M}^{3}\right)\right) \cong C\left(\pi_{1}\left(\mathcal{N}^{3}\right)\right) \cong \mathbb{Z}$. This implies that both $\mathcal{M}^{3}$ and $\mathcal{N}^{3}$ are Seifert manifolds $(c f .[\mathbf{C J}, \mathbf{G}])$. Let $h: \mathcal{M}^{3} \times \mathcal{N}^{3} \stackrel{\widetilde{\sim}}{\rightarrow} S_{1} \times S_{2} \times S^{1} \times S^{1}$ be a homeomorphism. The induced homomorphism

$$
h_{*}: \pi_{1}\left(\mathcal{M}^{3} \times \mathcal{N}^{3}\right) \rightarrow \pi_{1}\left(S_{1} \times S_{2} \times S^{1} \times S^{1}\right)
$$

is an isomorphism and

$$
h_{*}: C\left(\pi_{1}\left(\mathcal{M}^{3} \times \mathcal{N}^{3}\right)\right) \rightarrow C\left(\pi_{1}\left(S_{1} \times S_{2} \times S^{1} \times S^{1}\right)\right)
$$


is an isomorphism as well, i.e.,

$$
h_{* \mid}: \mathbb{Z} \oplus \mathbb{Z} \stackrel{\cong}{\longrightarrow} \oplus \mathbb{Z}
$$

To go further we resort to the following simple torus trick: Since every automorphism $\mathbb{Z} \oplus \mathbb{Z} \cong \mathbb{Z} \oplus \mathbb{Z}$ can be realized by a homeomorphism $S^{1} \times S^{1} \rightarrow S^{1} \times S^{1}$, that is, $\pi_{0}\left(\operatorname{Homeo}\left(T^{2}\right)\right) \cong \operatorname{GL}(2, \mathbb{Z})($ e.g., see Theorem 4 , p. 26 in $[\mathbf{R}])$. Then by composing

$$
h: \mathcal{M}^{3} \times \mathcal{N}^{3} \longrightarrow S_{1} \times S_{2} \times S^{1} \times S^{1}
$$

with $h^{\prime}=\operatorname{id}_{S_{1} \times S_{2}} \times f: S_{1} \times S_{2} \times S^{1} \times S^{1} \longrightarrow S_{1} \times S_{2} \times S^{1} \times S^{1}$ for some homeomorphism $f: S^{1} \times S^{1} \rightarrow S^{1} \times S^{1}$, we can assume that there is a homeomorphism

$$
h: \mathcal{M}^{3} \times \mathcal{N}^{3} \longrightarrow S_{1} \times S_{2} \times S^{1} \times S^{1}
$$

with the isomorphism $h_{* \mid}: \mathbb{Z} \oplus \mathbb{Z} \cong \mathbb{Z} \oplus \mathbb{Z}$ given by

$$
h_{* \mid}: h_{*}^{\prime} \oplus h_{*}^{\prime} .
$$

This implies that there is an induced homeomorphism

$$
\tilde{h}: \widetilde{\mathcal{M}^{3}} \times \widetilde{\mathcal{N}^{3}} \longrightarrow S_{1} \times S_{2} \times \mathbb{R} \times \mathbb{R},
$$

where $\widetilde{\mathcal{M}^{3}}, \widetilde{\mathcal{N}^{3}}$ are infinite cyclic coverings determined by the corresponding centers.

The fundamental groups $\pi_{1}\left(\widetilde{\mathcal{M}^{3}}\right) \cong \pi_{1}\left(\mathcal{M}^{3}\right) / C\left(\pi_{1}\left(\mathcal{M}^{3}\right)\right)$ and $\pi_{1}\left(\widetilde{\mathcal{N}^{3}}\right) \cong \pi_{1}\left(\mathcal{N}^{3}\right) /$ $C\left(\pi_{1}\left(\mathcal{N}^{3}\right)\right)$ are Fuchsian groups (since both $\mathcal{M}^{3}, \mathcal{N}^{3}$ are Seifert manifolds) and there is an isomorphism

$$
\pi_{1}\left(\widetilde{\mathcal{M}^{3}}\right) \times \pi_{1}\left(\widetilde{\mathcal{N}^{3}}\right) \cong \pi_{1}\left(S_{1}\right) \times \pi_{1}\left(S_{2}\right) .
$$

This implies (by Proposition II.37, p. 19, in $[\mathbf{J S}]$ ) that $\pi_{1}\left(\widetilde{M^{3}}\right)$ and $\pi_{1}\left(\widetilde{\mathcal{N}^{3}}\right)$ are isomorphic to fundamental groups of closed surfaces. It is not difficult to see (for example using the cohomology ring structure of closed surfaces) that corresponding groups must be isomorphic, say

$$
\pi_{1}\left(\widetilde{\mathcal{M}^{3}}\right) \cong \pi_{1}\left(S_{1}\right) \quad \text { and } \quad \pi_{1}\left(\widetilde{\mathcal{N}^{3}}\right) \cong \pi_{1}\left(S_{2}\right) .
$$

In particular,

$$
\widetilde{\mathcal{M}^{3}} \times \mathcal{N}^{3}=S_{1} \times S_{2} \times \mathbb{R} \times S^{1}
$$

and since $\pi_{1}\left(\widetilde{\mathcal{M}^{3}}\right) \cong \pi_{1}\left(S_{1}\right)$ then we have a homotopy equivalence

$$
S_{1} \times \mathcal{N}^{3} \simeq S_{1} \times S_{2} \times S^{1}
$$

and by symmetry

$$
\mathcal{M}^{3} \times \widetilde{\mathcal{N}^{3}}=S_{1} \times S_{2} \times S^{1} \times \mathbb{R}
$$

which gives

$$
\mathcal{M}^{3} \times S_{2} \simeq S_{1} \times S_{2} \times S^{1} .
$$

If one of the $S_{i}, i=1,2$ is $S^{2}$, say $S_{1}=S^{2}$, then $S^{2} \times \mathcal{N}^{3} \simeq S^{2} \times S_{2} \times S^{1}$ and

$$
\pi_{1}\left(\mathcal{N}^{3}\right) \cong \pi_{1}\left(S_{2} \times S^{1}\right) \cong \pi_{1}\left(S_{2}\right) \oplus \pi_{1}\left(S^{1}\right) .
$$

Consequently $\mathcal{N}^{3}=S_{2} \times S^{1}$ (cf. [He, p. 114]). In particular, we can assume $S_{1} \neq S^{2}$, $S_{2} \neq S^{2}$. 
Going back to the homotopy equivalence

$$
S_{1} \times \mathcal{N}^{3} \simeq S_{1} \times S_{2} \times S^{1}
$$

we observe that $S_{1} \times S_{2} \times S^{1}$ admits a metric (the standard product metric) of nonpositive curvature. The results of Farrell-Jones ( $c f$. $[\mathbf{F J}])$ imply $S_{1} \times \mathcal{N}^{3}=S_{1} \times\left(S_{2} \times\right.$ $\left.S^{1}\right)$. We claim that this is impossible given the indecomposability of $\mathcal{N}^{3}$.

To see this we need the following slight adjustment in the conclusion of $[\mathbf{K R} 1$, Theorem 1].

Claim 2.1. Let $X, Y$ be closed oriented surfaces of genus at least 2, and $\mathcal{N}^{3}$ be a Seifert manifold. If $\mathcal{N}^{3} \times X=\left(Y \times S^{1}\right) \times X$ then $\mathcal{N}^{3}=Y \times S^{1}$.

Proof of Claim 2.1. Let $\mathcal{M}^{3}=Y \times S^{1}$. Let $\beta \in \pi_{1}(X)$ be a fixed non-trivial element and $\alpha \in \pi_{1}\left(\mathcal{N}^{3}\right)$ be an arbitrary element. We recall $(c f .[\mathbf{T}])$ that the centralizer of each nontrivial element of $\pi_{1}(X)$ is an infinite cyclic subgroup.

Now the homeomorphism $g: \mathcal{N}^{3} \times X \longrightarrow \mathcal{M}^{3} \times X$ induces an isomorphism $g_{*}: \pi_{1}\left(\mathcal{N}^{3} \times X\right) \longrightarrow \pi_{1}\left(\mathcal{M}^{3} \times X\right)$ with $g_{*}(\alpha, 1)=\left(\alpha^{\prime}, \alpha^{\prime \prime}\right)$ and $g_{*}(1, \beta)=\left(\beta^{\prime}, \beta^{\prime \prime}\right)$.

Denote by $Z\left(\beta^{\prime \prime}\right)$ the centralizer of $\beta^{\prime \prime}$ and let $\gamma$ be its generator, i.e., $\langle\gamma\rangle \cong Z\left(\beta^{\prime \prime}\right)$.

Since $(\alpha, 1)(1, \beta)=(1, \beta)(\alpha, 1)$, then $\left(\alpha^{\prime}, \alpha^{\prime \prime}\right)\left(\beta^{\prime}, \beta^{\prime \prime}\right)=\left(\beta^{\prime}, \beta^{\prime \prime}\right)\left(\alpha^{\prime}, \alpha^{\prime \prime}\right)$. In particular, $\alpha^{\prime} \beta^{\prime}=\beta^{\prime} \alpha^{\prime}$ and $\alpha^{\prime \prime} \beta^{\prime \prime}=\beta^{\prime \prime} \alpha^{\prime \prime}$, and hence $\alpha^{\prime \prime} \in Z\left(\beta^{\prime \prime}\right)$. Therefore there exist integers $m, n$ such that $\alpha^{\prime \prime}=\gamma^{n}$ and $\beta^{\prime \prime}=\gamma^{m}$. Since $\pi_{1}\left(\mathcal{N}^{3}\right)$ is finitely generated (let us say by $\alpha_{1}, \ldots, \alpha_{k}$ with $\left.g_{*}\left(\alpha_{i}, 1\right)=\left(\alpha_{i}^{\prime}, \alpha_{i}^{\prime \prime}\right)\right)$ then $\alpha_{i}^{\prime \prime}=\gamma^{n_{i}}$ for some $i=1, \ldots, k$.

We claim that $\gamma^{n_{i}}=1$ for all $i=1, \ldots, k ;$ i.e., $n_{i}=0$, for $i=1,2, \ldots, k$.

To see this we argue by contradiction. Suppose the contrary. Then all $\gamma^{n_{i}}$ generate an infinite cyclic subgroup of $Z\left(\beta^{\prime \prime}\right)$, namely a subgroup generated by $\gamma^{r}$ where $r=$ $\operatorname{gcd}\left(n_{1}, \ldots, n_{k}\right)$. In this case we have an isomorphism

$$
\pi_{1}\left(\mathcal{N}^{3}\right) \cong g_{*}\left(\pi_{1}\left(\mathcal{N}^{3}\right), 1\right) \cong G \times Z,
$$

where $G \subseteq \pi_{1}\left(\mathcal{M}^{3}\right)$ and $Z \subseteq \pi_{1}(X)$. This forces $\mathcal{N}^{3}=Y \times S^{1}$ (cf. [He, p. 114]). Suppose then that all $n_{i}=0$. Then

$$
g_{*}\left(\pi_{1}\left(\mathcal{N}^{3}\right), 1\right) \subseteq \pi_{1}\left(\mathcal{M}^{3}\right) \times 1 .
$$

If the above inclusion is proper then

$$
\pi_{1}(X) \cong \pi_{1}\left(\mathcal{M}^{3}\right) / p_{1} g_{*}\left(\pi_{1}\left(\mathcal{N}^{3}\right), 1\right) \times \pi_{1}(X),
$$

where $p_{1}: \pi_{1}\left(\mathcal{M}^{3}\right) \times \pi_{1}(X) \longrightarrow \pi_{1}\left(\mathcal{M}^{3}\right)$ is the projection. This however is impossible. Consequently $g_{*}\left(\pi_{1}\left(\mathcal{N}^{3}\right), 1\right)=\pi_{1}\left(\mathcal{M}^{3}\right)$, and hence $\pi_{1}\left(\mathcal{N}^{3}\right) \cong \pi_{1}\left(\mathcal{M}^{3}\right)$ and $\mathcal{N}^{3}=$ $\mathcal{M}^{3}=Y \times S^{1}$.

This finishes the case when the genus of $S_{1}$ and $S_{2}$ is at least 2 .

Finally, if one of $S_{i}, i=1,2$ (or both) is a torus, then the center of one or both of $\pi_{1}\left(\mathcal{M}^{3}\right)$ and $\pi_{1}\left(\mathcal{N}^{3}\right)$ contains at least two copies of $\mathbb{Z}$. This implies that one or both of $\mathcal{M}^{3}, \mathcal{N}^{3}$ must be the torus $S^{1} \times S^{1} \times S^{1}$ and we are done. i.e.

Let us consider now the case of $\mathcal{M}^{3}$ and $\mathcal{N}^{2}$. Suppose $\mathcal{M}^{3} \times \mathcal{N}^{2}$ is decomposable,

$$
\mathcal{M}^{3} \times \mathcal{N}^{2}=S_{1} \times S_{2} \times S^{1}
$$

where $S_{1}, S_{2}$ are surfaces.

Our considerations are divided into two cases: 
(a) $\mathcal{N}^{2}=S^{2}$.

(b) $\mathcal{N}^{2}$ has genus $>1$.

Case (a): It follows that one of the $S_{i}, i=1,2$ must be $S^{2}$, say $S_{1}=S^{2}$. This gives $\mathcal{M}^{3} \times S^{2}=\left(S_{2} \times S^{1}\right) \times S^{2}$ and then $\mathcal{M}^{3}=S_{2} \times S^{1}$ because $\pi_{1}\left(\mathcal{M}^{3}\right) \cong \pi_{1}\left(S_{2}\right) \oplus \mathbb{Z}$. Case (b): In analogy with the case of $\mathcal{M}^{3}, \mathcal{N}^{3}$ it follows that, say $S_{1}=\mathcal{N}^{2}$ and hence

$$
\mathcal{M}^{3} \times \mathcal{N}^{2}=\left(S_{1} \times S^{1}\right) \times \mathcal{N}^{2}
$$

which implies $\mathcal{M}^{3}=S_{1} \times S^{1}$.

Finally we are left with the case $\mathcal{M}^{3}, \mathcal{N}^{1}$, so that $\mathcal{N}^{1}=S^{1}$. Then $\mathcal{M}^{3} \times S^{1}=$ $S_{1} \times S_{2}$. It follows that say $S_{1}=S^{1} \times S^{1}$ and we can assume $S_{2} \neq S^{1} \times S^{1}$. Indeed, if $S_{1}=S_{2}=S^{1} \times S^{1}$ then $\mathcal{M}^{3}=S^{1} \times S^{1} \times S^{1}$. Now

$$
\mathcal{M}^{3} \times S^{1}=\left(S^{1} \times S_{2}\right) \times S^{1} .
$$

Using the torus trick once again, we can arrange $\mathcal{M}^{3} \times \mathbb{R}=\left(S^{1} \times S_{2}\right) \times \mathbb{R}$ and hence $\pi_{1}\left(\mathcal{M}^{3}\right) \cong \pi_{1}\left(S_{2}\right) \oplus \mathbb{Z}$. This again implies $\mathcal{M}^{3}=S^{1} \times S_{2}$ contradicting the indecomposability of $\mathcal{M}^{3}$.

Proof of Theorem 1.2. In our proof we use a 4-manifold first constructed by Weinberger in [We] (see also [KS1, Theorem 2.1]). For completeness of this paper, we include a brief sketch of the construction with somewhat different reasoning. Let $\Sigma^{3}$ be a Seifert homology 3 -sphere with a natural free involution (i.e., free $\mathbb{Z}_{2}$-action) and Rochlin invariant $\mu\left(\Sigma^{3}\right)=1$. For example, we can let $\Sigma^{3}=\{\Sigma(5,7,11), \Sigma(3,5,13)$, $\Sigma(3,7,17), \Sigma(5,7,27)$ etc. ... $\}(c f .[\mathbf{N R}])$.

Now $\Sigma^{3} / \mathbb{Z}_{2}$ is a $\mathbb{Z}$-homology $\mathbb{R P}^{3}$, and there is a $\mathbb{Z}\left[\mathbb{Z}_{2}\right]$-homology equivalence (see $[\mathbf{K L}$, p. 35])

$$
f: \Sigma^{3} / \mathbb{Z}_{2} \longrightarrow \mathbb{R P}^{3}
$$

Let $I$ denote the interval $[0,1]$, and consider the map $h=f \times \operatorname{id}_{I}: \Sigma^{3} / \mathbb{Z}_{2} \times I \longrightarrow$ $\mathbb{R} P^{3} \times I$. This map $h$ is a $\mathbb{Z}\left[\mathbb{Z}_{2}\right]=\mathbb{Z}[\pi]$-homology equivalence. If $\Gamma_{0}(F)$, for $F=$ id: $\mathbb{Z}[\pi] \rightarrow \mathbb{Z}[\pi]$, is the Cappell-Shaneson homological surgery group (cf. [CS]) then obviously $\lambda(h)=0$ in $\Gamma_{0}(F)$, here $\lambda(h)$ is the surgery obstruction associated with $h$. But $L_{0}^{s, h}(\pi) \cong \Gamma_{0}(F)\left(c f\right.$. [CS, p. 289]) and hence $\lambda(h)=0$ in $L_{0}^{s, h}(\pi)$.

Consequently

$$
h: \Sigma^{3} / \mathbb{Z}_{2} \times I \longrightarrow \mathbb{R P}^{3} \times I
$$

is normally bordant to a homotopy equivalence (rel boundary). By identifying the corresponding boundaries (using the identity mapping) we obtain a homotopy equivalence $\mathcal{M} \longrightarrow \mathbb{R P}^{3} \times S^{1}$ which we shall also call $h$.

Claim 2.2. If $\widetilde{\mathcal{M}}$ is the infinite cyclic covering then there is no closed 3-manifold $\mathcal{N}^{3}$ with $\widetilde{\mathcal{M}}=\mathcal{N}^{3} \times \mathbb{R}$.

Proof of Claim 2.2. Suppose $\widetilde{\mathcal{M}}=\mathcal{N}^{3} \times \mathbb{R}$. Then there is no difficulty to see that there is a copy of $\Sigma^{3} / \mathbb{Z}_{2}$ far away in the $\mathbb{R}$-direction, which is disjoint with say $\mathcal{N}^{3} \times\{0\}$ in $\mathcal{N}^{3} \times \mathbb{R}$.

The region between $\mathcal{N}^{3} \times\{0\}$ and embedded $\Sigma^{3} / \mathbb{Z}_{2}$ is a homological $\mathbb{Z}\left[\mathbb{Z}_{2}\right] h$ cobordism $\left(\mathcal{W} ; \mathcal{N}^{3} ; \Sigma^{3} / \mathbb{Z}_{2}\right)$. Since $\mathcal{N}^{3}$ and $\Sigma^{3} / \mathbb{Z}_{2}$ are parallelizable there is a preferred spin structure on $\mathcal{N}^{3}$ and $\Sigma^{3} / \mathbb{Z}_{2}$. One can ask about the possibility of extending this 
structure to the entire $\mathcal{W}$. Whether one can do this or not is determined by the obstruction in $H^{4}\left(\mathcal{W} ; \partial \mathcal{W} ; \mathbb{Z}_{2}\right) \simeq \mathbb{Z}_{2}(c f .[\mathbf{K S 1}$, p. 448$])$.

There is an analogous obstruction for the existence of a spin structure on the 2fold cover $(\widetilde{\mathcal{W}} ; \partial \widetilde{\mathcal{W}})$. By the naturality, the obstruction in $H^{4}\left(\widetilde{\mathcal{W}} ; \partial \widetilde{\mathcal{W}} ; \mathbb{Z}_{2}\right) \simeq \mathbb{Z}_{2}$ is the image of the obstruction in $H^{4}\left(\mathcal{W} ; \partial \mathcal{W} ; \mathbb{Z}_{2}\right)$ under the homomorphism

$$
H^{4}\left(\mathcal{W} ; \partial \mathcal{W} ; \mathbb{Z}_{2}\right) \longrightarrow H^{4}\left(\widetilde{\mathcal{W}} ; \widetilde{\mathcal{W}} ; \mathbb{Z}_{2}\right)
$$

and hence the obstruction in $H^{4}\left(\widetilde{\mathcal{W}} ; \partial \widetilde{\mathcal{W}} ; \mathbb{Z}_{2}\right)$ is trivial. In other words the manifold $(\widetilde{\mathcal{W}} ; \partial \widetilde{\mathcal{W}})$ is quasi-spin in the terminology of [KS1, p. 449].

In our case $(\widetilde{\mathcal{W}} ; \partial \widetilde{\mathcal{W}})$ is a $\mathbb{Z}$-homological $h$-cobordism between $\Sigma^{3}$ and a homotopy 3 -sphere $\widetilde{\mathcal{N}^{3}}$. The Rochlin $\mu$-invariant is invariant with respect to topological quasispin $\mathbb{Z}$-homological- $h$-cobordism $[\mathbf{K S 1}]$ and hence $\mu\left(\widetilde{\mathcal{N}^{3}}\right)=\mu\left(\Sigma^{3}\right)$. However, this is a contradiction since $\mu\left(\Sigma^{3}\right)=1$ and $\mu\left(\widetilde{\mathcal{N}^{3}}\right)=0$ by the Casson's results (cf. [AM $]$ ), and hence Claim 2.2 has been established.

Now let $h: \mathcal{M} \rightarrow \mathbb{R P}^{3} \times S^{1}$ be the constructed homotopy equivalence.

Claim 2.3. The homotopy equivalence $h: \mathcal{M} \rightarrow \mathbb{R P}^{3} \times S^{1}$ is normally bordant to the identity.

Proof of Claim 2.3. Consider the Wall-Sullivan exact surgery sequence ( $c f$. [Wa]), which extends to dimension 4 by the results of $[\mathbf{F Q}]$ :

$$
\cdots \longrightarrow L_{1}^{s}\left(\mathbb{Z} \times \mathbb{Z}_{2}\right) \stackrel{\gamma}{\longrightarrow} S^{\mathrm{TOP}}\left(S^{1} \times \mathbb{R P}^{3}\right) \stackrel{\eta}{\longrightarrow}\left[S^{1} \times \mathbb{R P}^{3} ; G / \mathrm{TOP}\right] \stackrel{\lambda}{\longrightarrow} L_{0}^{s}\left(\mathbb{Z} \times \mathbb{Z}_{2}\right)
$$

Here $\left[S^{1} \times \mathbb{R P}^{3} ; G / \mathrm{TOP}\right] \cong H^{2}\left(S^{1} \times \mathbb{R P}^{3} ; \mathbb{Z}_{2}\right) \oplus H^{4}\left(S^{1} \times \mathbb{R} \mathbb{P}^{3} ; \mathbb{Z}\right) \cong \mathbb{Z}_{2} \oplus \mathbb{Z}_{2} \oplus \mathbb{Z}$.

By $[\mathbf{S h}]$ and the triviality of $\mathrm{Wh}\left(\mathbb{Z}_{2}\right)$ we have

$$
L_{1}^{s}\left(\mathbb{Z} \times \mathbb{Z}_{2}\right) \cong L_{1}^{s}\left(\mathbb{Z}_{2}\right) \oplus L_{0}^{s}\left(\mathbb{Z}_{2}\right)
$$

and

$$
L_{0}^{s}\left(\mathbb{Z} \times \mathbb{Z}_{2}\right) \cong L_{0}^{s}\left(\mathbb{Z}_{2}\right) \oplus L_{3}^{s}\left(\mathbb{Z}_{2}\right) \cong L_{0}^{s}(0) \oplus \widetilde{L_{0}^{s}}\left(\mathbb{Z}_{2}\right) \oplus \mathbb{Z}_{2}
$$

in which $L_{0}^{s}(0)$ and $\widetilde{L_{0}^{s}}\left(\mathbb{Z}_{2}\right)$ are both isomorphic to $\mathbb{Z}$.

Let us briefly analyze the subgroup $\operatorname{Ooze}\left(\mathbb{Z} \times \mathbb{Z}_{2}\right) \subset L_{0}^{s}\left(\mathbb{Z} \times \mathbb{Z}_{2}\right)$. We recall that the Ooze $(-)$ subgroup is represented by surgery obstructions determined by closed manifolds ( $c f .[$ HMTW]).

It turns out that $\operatorname{Ooze}\left(\mathbb{Z} \times \mathbb{Z}_{2}\right) \cong L_{0}^{s}(0) \oplus L_{3}^{s}\left(\mathbb{Z}_{2}\right) \cong \mathbb{Z} \oplus \mathbb{Z}_{2}$. To see this, just note that $\mathbb{Z} \cong L_{0}^{s}(0)$ is represented by the difference of signatures and the existence of the $E_{8}$ manifold $(c f .[\mathbf{F Q}])$ implies $L_{0}^{s}(0) \subset \operatorname{Ooze}\left(\mathbb{Z} \times \mathbb{Z}_{2}\right)$.

The copy of $\mathbb{Z}_{2} \cong L_{3}^{s}\left(\mathbb{Z}_{2}\right)$ is determined by the codimension one Arf invariant (cf. $\left[\right.$ HMTW, Theorem A]) in $\left[\mathbb{R P}^{3} ; \mathbb{Z}_{2}\right] \stackrel{\lambda}{\longrightarrow} L_{3}^{s}\left(\mathbb{Z}_{2}\right)$.

In fact, $\mathbb{Z}_{2} \cong\left[\mathbb{R P}^{3} ; G / \mathrm{TOP}\right] \cong H^{2}\left(\mathbb{R P}^{3} ; \mathbb{Z}_{2}\right)$ corresponds to the copy of $\mathbb{Z}_{2} \subset$ $H^{2}\left(S^{1} \times \mathbb{R} \mathbb{P}^{3} ; \mathbb{Z}_{2}\right)$ given by $H^{0}\left(S^{1} ; \mathbb{Z}_{2}\right) \otimes H^{2}\left(\mathbb{R} \mathbb{P}^{3} ; \mathbb{Z}_{2}\right)$.

The remaining copy of $\mathbb{Z}_{2} \subset H^{2}\left(S^{1} \times \mathbb{R P}^{3} ; \mathbb{Z}_{2}\right)$ corresponds to $H^{1}\left(S^{1} ; \mathbb{Z}_{2}\right) \otimes$ $H^{1}\left(\mathbb{R P}^{3} ; \mathbb{Z}_{2}\right)$. 
It turns out that this remaining copy of $\mathbb{Z}_{2}$ is represented by a self-homotopy equivalence

$$
s: S^{1} \times \mathbb{R P}^{3} \longrightarrow S^{1} \times \mathbb{R P}^{3}
$$

given by the pinching construction. To be more specific the self-homotopy equivalence $s$ is given by

$$
s: S^{1} \times \mathbb{R}^{3} \stackrel{\vee}{\longrightarrow} S^{1} \times \mathbb{R P}^{3} \vee S^{4} \stackrel{\operatorname{id} \vee v}{\longrightarrow} S^{1} \times \mathbb{R P}^{3},
$$

where $v: S^{4} \rightarrow S^{1} \times \mathbb{R} \mathbb{P}^{3}$ is the nontrivial homotopy class of $\pi_{4}\left(S^{3}\right) \cong \mathbb{Z}_{2}$.

The homotopy theoretic argument for this is given in [KS2, Theorem 2.1, Case II, p. 531].

The argument in $[\mathbf{K S 2}]$ is given for a self-homotopy equivalence (rel boundary) of $\mathbb{R} \mathbb{P}^{3} \times I$, but it works in precisely the same way for $S^{1} \times \mathbb{R} \mathbb{P}^{3}$.

A consequence of the above considerations is that the homotopy (simple) equivalence

$$
h: \mathcal{M} \longrightarrow S^{1} \times \mathbb{R P}^{3}
$$

being an element of $S^{\mathrm{TOP}}\left(S^{1} \times \mathbb{R P}^{3}\right)$ must be normally bordant to the identity.

(We recall that $\mathrm{Wh}\left(\mathbb{Z} \times \mathbb{Z}_{2}\right) \cong \mathrm{Wh}\left(\mathbb{Z}_{2}\right) \oplus \widetilde{K_{0}}\left(\mathbb{Z}\left[\mathbb{Z}_{2}\right]\right) \cong 0$; see $[\mathbf{B H S}]$ and $[\mathbf{H a}]$.)

Let $\left(\mathcal{W} ; \mathcal{M}, S^{1} \times \mathbb{R P}^{3}\right)$ be the corresponding normal bordism:

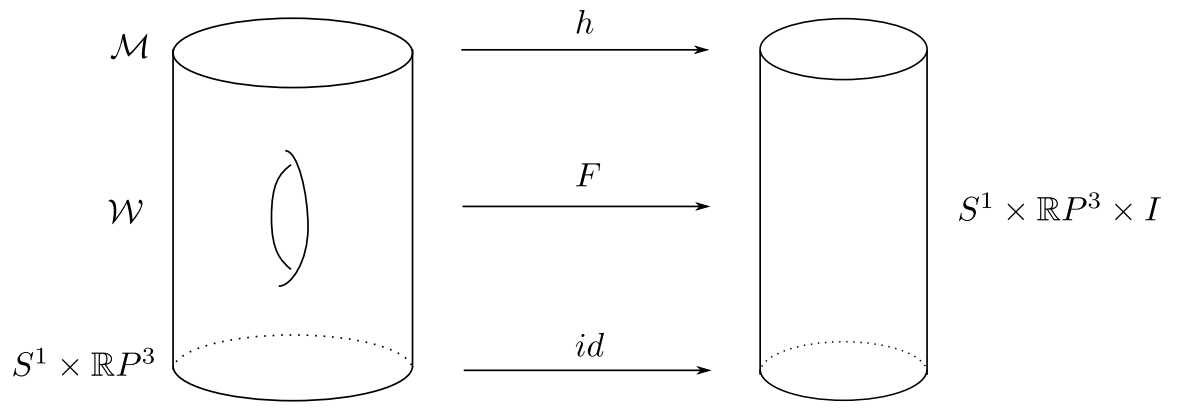

Figure 1: The normal cobordism $\left(\mathcal{W} ; \mathcal{M}, S^{1} \times \mathbb{R P}^{3}\right)$.

Multiplying the above normal bordism by $\operatorname{id}_{S^{i}}: S^{i} \rightarrow S^{i}(i=2,3,4)$ we get the surgery obstruction $(c f .[\mathbf{M}])$ :

$$
\lambda\left(F \times \operatorname{id}_{S^{i}}\right)=\lambda(F) \cdot \sigma^{*}\left(S^{i}\right)=0 .
$$

In particular, $S^{1} \times \mathbb{R P}^{3} \times S^{i}$ is s-cobordant to $\mathcal{M} \times S^{i}$ and hence $\mathcal{M} \times S^{i}=S^{1} \times$ $\mathbb{R P}^{3} \times S^{i}$

This finishes the proof of Theorem 1.2, once we know that the manifold $\mathcal{M}$ is indecomposable. This however follows from the Claim 2.2. We are then left with the construction of infinitely many corresponding examples.

To do this we follow $[\mathbf{K S 1}$. Consider the extension of the Wall-Sullivan exact sequence to dimensional 3 (cf. $[\mathbf{J K}])$ :

$$
\cdots \longrightarrow L_{0}^{s}\left(\mathbb{Z}_{2}\right) \longrightarrow S^{H}\left(\mathbb{R P}^{3}\right) \longrightarrow\left[\mathbb{R P}^{3} ; G / \mathrm{TOP}\right] \longrightarrow L_{3}^{s}\left(\mathbb{Z}_{2}\right) \text {. }
$$

Now $L_{0}^{s}\left(\mathbb{Z}_{2}\right) \cong L_{0}^{s}(0) \oplus \widetilde{L_{0}^{s}}\left(\mathbb{Z}_{2}\right) \cong \mathbb{Z} \oplus \mathbb{Z}$ and $\widetilde{L_{0}^{s}}\left(\mathbb{Z}_{2}\right)$ acts freely on $S^{H}\left(\mathbb{R P}^{3}\right)$. This implies 
(a) the existence of infinitely many homology 3 -spheres $\left(\Sigma_{i}^{3}, t_{i}\right), i=1,2, \ldots$ with $\mu\left(\Sigma_{i}^{3}\right)=1$ and a free involution $t_{i}: \Sigma_{i} \rightarrow \Sigma_{i}$

(b) the $\rho$-invariant associated with these actions are different, i.e. $\rho\left(\Sigma_{i}^{3}, t_{i}\right)-$ $\rho\left(\Sigma_{j}^{3}, t_{j}\right) \neq 0$ for $i \neq j$.

The crucial fact needed here is the congruence

$$
\mu\left(\Sigma_{i}^{3}\right) \equiv \rho\left(\Sigma_{i}^{3}, t_{i}\right) \bmod 16
$$

(cf. $[\mathbf{N R}])$.

Given the above we start with $\Sigma_{i}^{3} / \mathbb{Z}_{2} \times I$ and convert it by topological surgery to a homotopy equivalence

$$
\left(\overline{\mathcal{W}_{i}}, \partial\right) \longrightarrow\left(\mathbb{R P}^{3} \times I, \partial\right)
$$

which is a $\mathbb{Z}\left[\mathbb{Z}_{2}\right]$-homology equivalence of boundaries.

Next form a two ended open manifold $\widetilde{\mathcal{W}}_{i}$ by taking infinitely many copies of $\overline{\mathcal{W}_{i}}$, one on the top of the another.

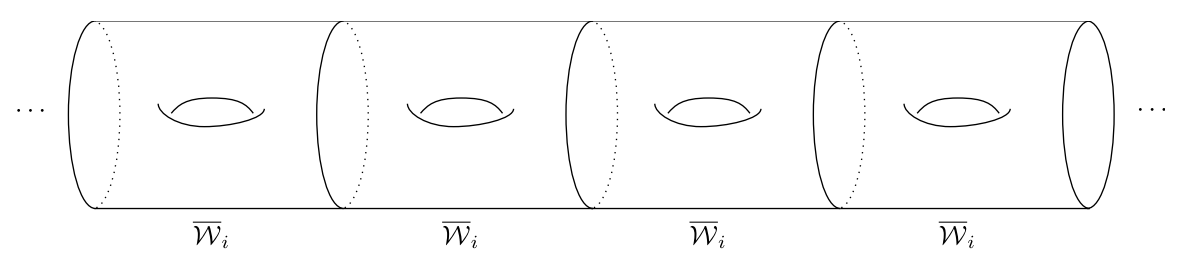

Figure 2: Open manifold $\widetilde{\mathcal{W}}_{i}$.

There is a natural free proper action of $\mathbb{Z}$ on $\widetilde{\mathcal{W}_{i}}$ and we shall let

$$
\mathcal{W}_{i}:=\widetilde{\mathcal{W}}_{i} / \mathbb{Z}
$$

The manifold $\mathcal{W}_{i}(i=1,2, \ldots)$ has the required properties, more precisely, we have the following:

(1) $\mathcal{W}_{i} \simeq S^{1} \times \mathbb{R P}^{3}(i=1,2, \ldots)$.

(2) $\mathcal{W}_{i}$ is indecomposable.

(3) $\mathcal{W}_{i} \neq \mathcal{W}_{j}, i \neq j$.

(4) $\mathcal{W}_{i} \times S^{k}=S^{1} \times \mathbb{R P}^{3} \times S^{k}(k=2,3,4)$.

Proof of Theorem 1.3. Suppose $\mathcal{M}^{4} \times S^{4}$ is decomposable. Then there are two cases:

(a) $\mathcal{M}^{4} \times S^{4}=S^{2} \times K^{6}$

(b) $\mathcal{M}^{4} \times S^{4}=\Sigma^{3} \times K^{5}$

for some closed manifolds $K^{6}$ and $K^{5}$, and a homotopy 3-sphere $\Sigma^{3}$.

The case (b) cannot occur because $\chi\left(\mathcal{M}^{4} \times S^{4}\right) \neq 0$ and $\chi\left(\Sigma^{3} \times K^{5}\right)=0$.

Consider now case (a). Note that one can assume $H^{2}\left(\mathcal{M}^{4}\right) \neq 0, \mathbb{Z}$. Suppose then that $H^{2}\left(\mathcal{M}^{4}\right) \cong \mathbb{Z} \oplus \mathbb{Z}$. Then

$$
H^{2}\left(\mathcal{M}^{4} \times S^{4}\right) \cong H^{2}(\mathcal{M}) \cong H^{2}\left(S^{2}\right) \oplus H^{2}\left(K^{6}\right) .
$$

It follows that the matrix for the standard intersection form on $H^{2}(\mathcal{M})$ is given by 
$\left(\begin{array}{ll}0 & 1 \\ 1 & 0\end{array}\right)$. This however implies $\mathcal{M}=S^{2} \times S^{2}$ which is a contradiction.

Next, suppose $H^{2}\left(\mathcal{M}^{4}\right) \cong \underset{r}{\oplus} \mathbb{Z}$, where $r \geqslant 3$. In this case the matrix for the intersection form on $H^{2}\left(\mathcal{M}^{4}\right)$ is given by

$$
\left(\begin{array}{ccccc}
0 & 1 & 1 & \cdots & 1 \\
1 & 0 & 0 & \cdots & 0 \\
1 & 0 & 0 & & \\
\vdots & \vdots & & \ddots & \\
1 & 0 & & & 0
\end{array}\right) .
$$

This again is a contradiction because the determinant of the displayed matrix is zero.

The case of $\mathcal{M}^{4} \times S^{3}$ is easier. The only possible decomposition of $\mathcal{M}^{4} \times S^{3}$ is given by $\mathcal{M}^{4} \times S^{3}=\Sigma^{3} \times S^{2} \times S^{2}$ for a homotopy 3 -sphere $\Sigma^{3}$.

Finally, $\mathcal{M}^{4} \times S^{2}$ can only be decomposed as $\mathcal{M}^{4} \times S^{2}=S^{2} \times S^{2} \times S^{2}$.

\section{Acknowledgments}

We are grateful to Professor Witold Rosicki for numerous discussions and suggestions which have improved this paper in several ways.

\section{References}

[AM] S. Akbulut and J. McCarthy, Casson's invariant for oriented homology 3-spheres: an exposition, Princeton University Press, Princeton, 1990.

[BHS] H. Bass, A. Heller and R. Swan, The Whitehead group of polynomial extension, Publ. Math. Inst. Hautes Études Sci. 22 (1964), 35-63.

[Bi] R.H. Bing, The cartesion product of a certain non-manifold and a line is $E^{4}$, Ann. of Math. 70 (1959), 399-412.

[Bo] K. Borsuk, On decomposition of manifolds into products of curves and surfaces, Fund. Math. 33 (1945), 273-298.

[CJ] A. Casson and D. Jungreis, Convergence groups and Seifert fibered 3manifolds, Invent. Math. 118 (1994), 441-456.

[CR] P. Conner and F. Raymond, Derived actions, in Proc. Second Conf. Compact Transf. Groups, Amherst, 1971, in: Lecture Notes in Math., Vol. 299, Springer, Berlin/New York, 1972, pp. 237-310.

[CS] S. Cappell and J. Shaneson, The codimension two placement problem and homology equivalent manifolds, Ann. of Math. 99 (1974), 277-348.

[FJ] F.T. Farrell and L.E. Jones, Topological rigidity for compact nonpositively curved manifolds, Proc. Sympos. Pure Math. 54 Part 3 (1993), pp. 229-274.

[FQ] M. Freedman and F. Quinn: topology of 4-manifolds, Princeton Math. Ser. 39, Princeton Univ. Press, Princeton, 1990.

[G] D. Gabai, Convergence groups are Fuchsian groups, Ann. of Math. 136 (1992), 447-510. 
[Ha] D.R. Harmon, $N K_{1}$ of finite groups, Proc. Amer. Math. Soc., Vol. 100, No. 2 (1987) 229-232.

[He] J. Hempel, 3-manifolds, Ann. of Math. Stud., No. 86, Princeton Univ. Press, Princeton, 1976.

[HMTW] I. Hambleton, R.J. Milgram, L. Taylor and B. Williams, Surgery with finite fundamental group, Proc. Lond. Math. Soc. (3) 56 (1988), 349-379.

[JK] B. Jahren and S. Kwasik, Three-dimensional surgery theory, UNil-groups and the Borel Conjecture, Topology 42 (2003), 1353-1369.

[JS] W.H. Jaco and P. Shalen, Seifert fibered spaces in 3-manifolds, Mem. Amer. Math. Soc., Vol. 21, No. 220 (1979).

[KL] S. Kwasik and T. Lawson, Nonsmoothable $\mathbb{Z}_{p}$ actions on contractible 4-manifolds, J. Reine Angew. Math. 437 (1993), 29-54.

[KR1] S. Kwasik and W. Rosicki, Cartesian product stabilization of 3manifolds, Topology Appl. 157 (2010), 2342-2346.

[KR2] S. Kwasik and W. Rosicki, On stability of 3-manifolds, Fund. Math. 182 (2004), 169-180.

[KS1] S. Kwasik and R. Schultz, Desuspension of group actions and the Ribbon Theorem, Topology 27 (1988), 443-457.

[KS2] S. Kwasik and R. Schultz, On $s$-cobordisms of metacyclic prism manifolds, Invent. Math. 97 (1989), 523-552.

[M] J. Morgan, A product formula for surgery obstructions, Mem. Amer. Math. Soc., Vol. 14, No. 201, AMS, 1978.

[NR] W. Neumann and F. Raymond, Seifert manifolds, plumbing $\mu$-invariant and orientation reversing maps, Lecture Notes in Math. 664, pp. 163169, Springer-Verlag, 1978.

[R] D. Rolfsen, Knots and Links, Math. Lecture Ser., Vol. 7, Publish or Perish, Berkeley, 1976.

[Sh] J. Shaneson, Wall's surgery obstruction group for $\mathbb{Z} \times G$, Ann. of Math. 90 (1969), 296-334.

[T] B. Truffault, Centralisateurs des elements dans les groups de Greendlinger. C. R. Acad. Sci. Paris, Ser. A. 279 (1974), pp. 317-319.

[Wa] C.T.C. Wall, Surgery on Compact Manifolds, London Math. Soc. Monogr. Ser., No. 1, Academic Press, London and New York, 1970.

[We] S. Weinberger, On fibering four- and five-manifolds, Israel J. Math. 59 (1987), 1-7.

Slawomir Kwasik kwasik@tulane.edu

Department of Mathematics, Tulane University, New Orleans, LA 70118, USA

Reinhard Schultz schultz@math.ucr.edu

Department of Mathematics, University of California, Riverside, CA 92521, USA 